\title{
GEOESTATÍSTICA NA AVALIAÇÃO DOS ATRIBUTOS FÍSICOS EM LATOSSOLO SOB FLORESTA NATIVA E PASTAGEM NA REGIÃO DE MANICORÉ, AMAZONAS ${ }^{(1)}$
}

\author{
Renato Eleotério de Aquino ${ }^{(2)}$, Milton César Costa Campos ${ }^{(3)}$, José Marques Júnior ${ }^{(4)}$ Ivanildo \\ Amorim de Oliveira ${ }^{(2)}$, Bruno Campos Mantovaneli ${ }^{(5)}$ \& Marcelo Dayron Rodrigues Soares ${ }^{(6)}$
}

\section{RESUMO}

A utilização das técnicas geoestatísticas permite detectar a existência da dependência e distribuição espacial dos atributos do solo, constituindo importante ferramenta na análise e descrição detalhada do comportamento dos atributos físicos do solo. Este trabalho teve como objetivo o uso da geoestatística na avaliação dos atributos físicos em Latossolo sob floresta nativa e pastagem na região de Manicoré no Amazonas. Nas áreas de floresta nativa e pastagem, foram estabelecidas malhas com dimensão $70 \times 70 \mathrm{~m}$ e demarcados pontos nessas malhas espaçados a cada $10 \mathrm{~m}$, totalizando 64 pontos. Esses pontos foram georreferenciados e, em seguida, foram feitas as coletadas de solo em cada ponto da malha nas camadas de 0,00-0,20 e 0,40-0,60 m para determinação dos atributos físicos, totalizando 128 amostras de solo em cada malha. Essas malhas encontram-se paralelas com uma distância uma da outra de $100 \mathrm{~m}$ e o solo nessas áreas é classificado como Latossolo. Determinaram-se textura, densidade do solo e de partículas, macroporosidade, microporosidade, porosidade total e estabilidade dos agregados em água. Após a tabulação dos dados, foram realizadas análises estatísticas descritivas e geoestatística. A pastagem apresentou leve variação nos seus atributos físicos em relação à floresta nativa, com coeficiente de variação alto e dependência espacial fraca. Os semivariogramas escalonados conseguiram reproduzir de forma satisfatória o comportamento espacial dos atributos no mesmo padrão dos semivariogramas individuais, e o uso do parâmetro alcance do semivariograma mostrou-se eficiente para determinar a densidade amostral ideal para os ambientes

(1) Projeto de Pesquisa financiado pela FAPEAM e SECT-AM. Recebido para publicação em 16 de julho de 2013 e aprovado em 20 de novembro de 2013.

(2) Doutorandos da Faculdade de Ciências Agrárias e Veterinárias, UNESP. Via de Acesso Prof. Paulo Donato Castellane. CEP 14870-900, Jaboticabal (SP), Brasil. E-mail: aquino.rea@gmail.com, ivanildoufam@gmail.com

(3) Prof. Adjunto II, Instituto de Educação, Agricultura e Ambiente, UFAM. Rua 29 de Agosto, 786, Centro. CEP 69800-000 Humaitá (AM), Brasil. E-mail: mcesarsolos@gmail.com

(4) Prof. do Departamento de Solos e Adubos, FCAV/UnESP, Via de Acesso Prof. Paulo Donato Castellane. CEP 14870-900 Jaboticabal (SP), Brasil. E-mail: marques@fcav.unesp.br

(5) Acadêmico de Agronomia do Instituto de Educação, Agricultura e Ambiente - IEAA-UFAM. E-mail: brunomantovanelli21@gmail.com

(6) Mestrando da Universidade Federal do Acre - UFAC, Programa de Pós-graduação em Ciência, Inovação e Tecnologia para a Amazônia - CITA. Rod. 364, Km 04, B, Distrito Industrial. CEP 69915-900 Rio Branco (AC), Brasil. E-mail: marcelo.dayron@gmail.com 
em estudo. Os resultados geoestatísticos indicaram que a retirada da floresta nativa para a implantação da pastagem alterou a variabilidade natural dos atributos físicos.

Termos de indexação: geoestatística, dependencia espacial, densidade amostral, sul do Amazonas.

\title{
SUMMARY: GEOSTATISTICS IN ASSESSMENT OF PHYSICAL PROPERTIES IN A LATOSSOLO (OXISOL) UNDER NATIVE FOREST AND GRASSLAND IN MANICORÉ, AMAZONAS, BRAZIL
}

\begin{abstract}
The use of geostatistical techniques allows detection of the existence of dependence and the spatial distribution of soil properties, thus constituting an important tool in the analysis and detailed description of the behavior of soil physical properties. The aim of the present study was to use geostatistics in assessment of physical properties in a Latossolo (Oxisol) dystrophic under native forest and pasture in the Amazon region of Manicore. Grids with of $70 \times 70 \mathrm{~m}$ were established in native forest and pasture, and points were marked in these grids spaced at every $10 \mathrm{~m}$, for a total of 64 points. These points were then georeferenced and in each one, soil samples (128) were collected at the depths of 0.00-0.20 and 0.40-0.60 $\mathrm{m}$ for a survey of their physical properties. These grids are parallel at a distance of $100 \mathrm{~m}$ from one another. The following determinations were made: texture, bulk density and particle density, macroporosity, microporosity, total porosity and aggregate stability in water. After tabulating the data, descriptive statistical analysis and geostatistical analysis were performed. The pasture had a slight variation in its physical properties in relation to native forest, with a high coefficient of variation and weak spatial dependence. The scaled semivariograms were able to satisfactorily reproduce the spatial behavior of the properties in the same pattern as the individual semivariograms, and the use of the parameter range of the semivariogram was efficient for determining the optimal sampling density for the environments under study. The geostatistical results indicate that the removal of native forest for establishing pasture altered the natural variability of the physical properties.
\end{abstract}

Index terms: geostatistics, spatial dependence, sampling density, southern Amazon.

\section{INTRODUÇÃO}

Historicamente, as áreas de maior concentração de desmatamento na Amazônia estão localizadas ao longo do arco do desmatamento, que faz parte a região sul do Amazonas (Becker, 2005). O desmatamento nessa região é um fenômeno atribuído a diversos fatores, como exploração agrícola, pecuária e principalmente florestal (Alencar et al., 2004). Na medida em que o uso e a ocupação ocorrem sem a preocupação com um manejo adequado dessas áreas, verifica-se crescente degradação dos recursos naturais (Lima et al., 2009).

Nesse contexto, os solos da região amazônica, onde predominam os Latossolos e Argissolos, apresentam fertilidade natural muito baixa (Cardoso et al., 2009). Esses ambientes amazônicos vêm sofrendo alterações dos ecossistemas naturais por meio da retirada da cobertura vegetal, o que tem promovido o rompimento do equilíbrio entre solo e o meio, modificando seus atributos químicos, físicos e biológicos, limitando sua utilização sustentável (Lima et al., 2009).

Assim, o conhecimento da distribuição espacial dos atributos do solo em uma área que teve conversão de floresta para pastagem é de extrema importância, pois os processos de formação que imprimiram, ao longo do tempo, variabilidade e essa, somada ao manejo realizado pelo homem, acentuam a variação dos atributos do solo (Cavalcante et al., 2007); seu entendimento é fundamental para o refinamento das práticas de manejo e avaliação dos efeitos sobre o ambiente (Cambardella et al., 1994).

O estudo da variabilidade espacial por meio da geoestatística possibilita a interpretação dos resultados com base na estrutura da variabilidade natural dos atributos avaliados, considerando a dependência espacial dentro do intervalo de amostragem (Souza et al., 2009), permitindo indicar alternativas de uso, além de possibilitar melhor compreensão da variabilidade dos atributos e sua influência no ambiente (Silva Neto et al., 2012).

Uma das grandes dificuldades encontradas nos estudos de variabilidade espacial dos atributos do solo tem sido a determinação do espaçamento ideal de amostragem, o que, em algumas situações, inviabiliza a adoção das técnicas da agricultura de precisão (Montanari et al., 2005; Souza et al., 2006a,b). O estudo dos aspectos da amostragem do solo, a fim de subsidiar definições sobre a utilização e recomendação dessas técnicas em ambientes distintos, tem sido preocupação constante de pesquisadores (Lark, 2000; Montanari et al., 2005; Souza et al., 2006a,b; Coelho et al., 2009).

Em relação aos atributos físicos do solo, a maioria dos estudos tem demonstrado ampla diversidade de resultados, com variação entre os locais estudados com 
ou sem continuidade espacial, provavelmente associada ao sistema de manejo e às características intrínsecas dos solos (Grego \& Vieira, 2005). Os atributos físicos do solo são bons indicadores de qualidade e permitem o monitoramento de áreas que sofreram algum tipo de interferência.

Estudos na região sul do Amazonas, feitos por Campos et al. (2012; 2013a,b) e Oliveira et al. (2013), sobre atributos físicos do solo em áreas com plantio de mandioca, cana-de-açúcar e agrofloresta permitiram melhor compreensão dos atributos estudados nesses locais com a aplicação da geoestatística. Diante disso, o objetivo deste trabalho foi aplicar a geoestatística na avaliação dos atributos físicos em Latossolo sob floresta nativa e pastagem na região de Manicoré, Amazonas.

\section{MATERIAL E MÉTODOS}

O estudo foi realizado em uma propriedade rural, localizada ao sul do Estado do Amazonas, nas imediações da comunidade Santo Antônio de Matupí, às margens da $\mathrm{BR} 230$, na região de Manicoré, AM. A área de floresta está situada nas coordenadas geográficas $7^{\circ} 54^{\prime} 44,5^{\prime \prime} \mathrm{S}$ e $61^{\circ} 31^{\prime} 44,7^{\prime \prime} \mathrm{W}$, com altitude média de $140 \mathrm{~m}$. Paralela à área de floresta, encontra-se situada a área de pastagem sob as coordenadas geográficas: $07^{\circ} 54^{\prime} 42^{\prime \prime} \mathrm{S}$ e $61^{\circ} 31^{\prime} 50^{\prime \prime} \mathrm{W}$, com altitude média de 135 m (Figura 1).
O material de origem é proveniente da alteração de granitos Rondonianos, do Pré-Cambriano Superior, sedimentos coluviais, depositados nas partes mais baixas da paisagem e coberturas terciárias (Brasil, 1978). O clima da região, segundo a classificação de Köppen, é do tipo tropical chuvoso, com um período seco de pequena duração do tipo Am, temperaturas variando entre 25 e $27^{\circ} \mathrm{C}$ e precipitações pluviais entre 2.250 e $2.750 \mathrm{~mm}$, com chuvas concentradas no período de outubro a junho (Brasil, 1978).

Nessas áreas, foram estabelecidas malhas com dimensão $70 \times 70 \mathrm{~m}$ e demarcados pontos nessas malhas, espaçados a cada $10 \mathrm{~m}$, totalizando 64 pontos, conforme figura 1. Esses pontos foram georreferênciados com um equipamento de GPS Garmin Etrex (South American'69). Em seguida, foram feitas as coletadas de solo em cada ponto da malha nas camadas de $0,00-0,20$ e 0,40-0,60 m para determinação dos atributos físicos (análise granulométrica, macro e microporosidade, porosidade total, densidade do solo e de partículas, e estabilidade de agregados), totalizando 128 amostras de solo em cada malha.

Essas malhas encontram-se paralelas com uma distância uma da outra de 100 m, e o solo nessas áreas foi classificado como Latossolo (Embrapa, 2006). A área de floresta nativa encontra-se intacta, mantida como área de preservação na propriedade rural; a área sob pastagem já é explorada por 10 anos, sendo essa usada somente para a pastagem. Durante todo esse tempo de uso não foi realizado nenhum tipo de preparo do solo para a manutenção de sua qualidade.

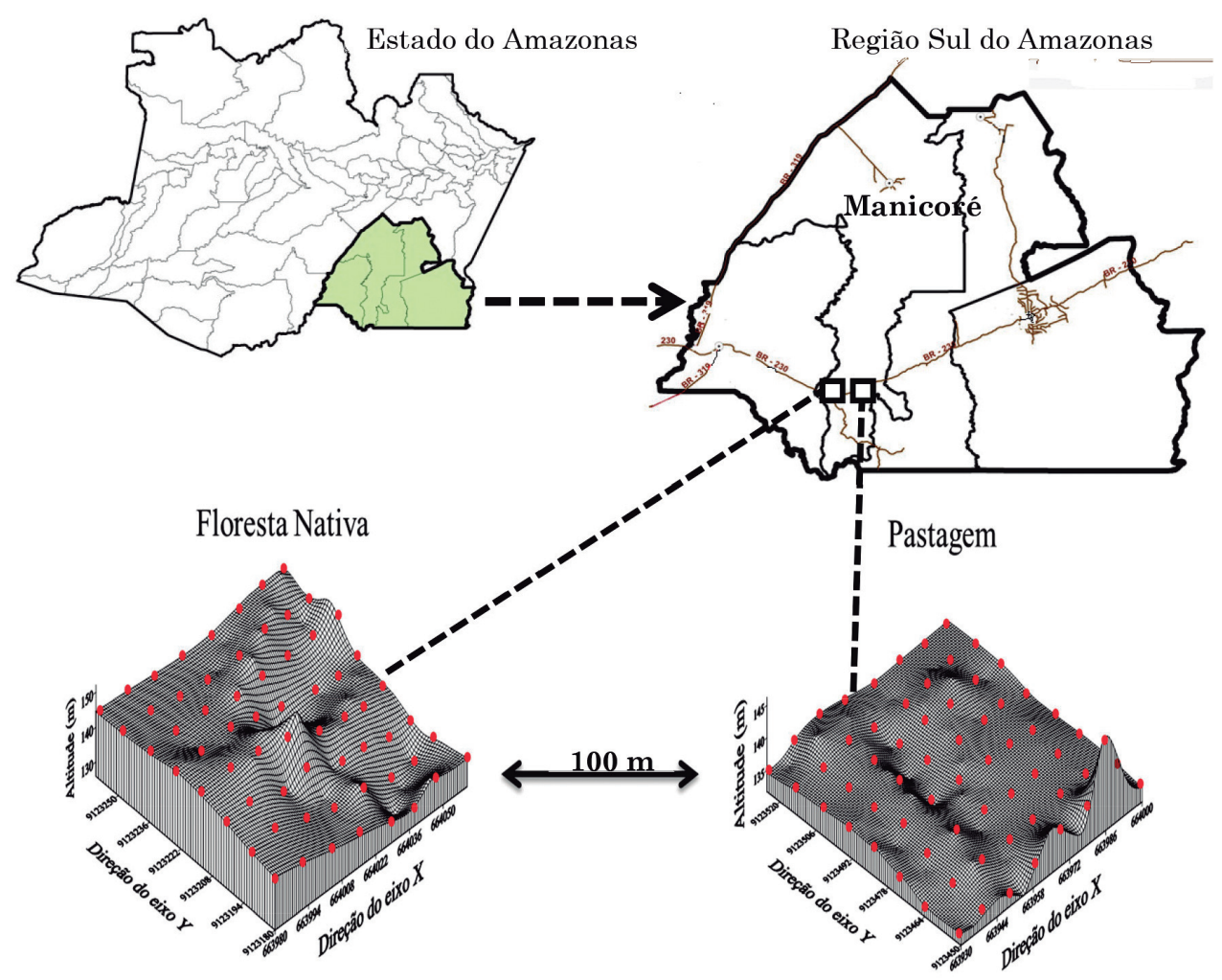

Figura 1. Localização da área de estudo, modelo de elevação digital e pontos de coleta de amostras de solo. 
A análise granulométrica foi realizada pelo método da pipeta na camada de $0,0-0,20$ e $0,40-0,60 \mathrm{~m}$, utilizando solução de $\mathrm{NaOH} 0,1 \mathrm{~mol} \mathrm{~L}^{-1}$ como dispersante químico e agitação mecânica em aparato de alta rotação ( 15.000 rpm) por $15 \mathrm{~min}$, seguindo método proposto pela Embrapa (1997).

As demais análises físicas foram realizadas somente na camada de $0,0-0,20 \mathrm{~m}$. As coletas de solo com estrutura preservada foram realizadas por meio de anel volumétrico com 0,045 m de altura e 0,045 m de diâmetro. Em seguida, foram saturadas por meio da elevação gradual de uma lâmina de água até atingir cerca de $2 / 3$ da altura do anel, determinando assim a porosidade total, a microporosidade, macroporosidade e a densidade do solo (Embrapa, 1997). A densidade de partícula foi definida pelo método do balão volumétrico, conforme método da Embrapa (1997).

Para especificação da distribuição de agregados, foram coletados blocos de solo com estrutura preservada secos ao ar e passados em peneiras de 9,52 e 4,76 $\mathrm{mm}$. Os agregados retidos na peneira de 4,76 $\mathrm{mm}$ foram empregados na análise de estabilidade de agregados via úmida, segundo Kemper \& Chepil (1965), que foi realizada colocando as amostras sobre um jogo de peneiras com malhas de 2,$0 ; 1,0 ; 0,5 ; 0,25$; 0,125 ; e $0,063 \mathrm{~mm}$ e submetendo-as a oscilações verticais durante $15 \mathrm{~min}$, numa frequência de 32 oscilações por min. Foram adotados como índices de estabilidade, os agregados nas classes $>2,2-1$ e $<1$ $\mathrm{mm}$, o diâmetro médio geométrico (DMG) e o diâmetro médio ponderado (DMP), cujos cálculos foram feitos segundo Kemper \& Rosenau (1986).

Após obtenção dos dados, foi avaliada a sua variabilidade primeiramente, pela análise exploratória, calculando-se a média, a mediana, o desvio-padrão, a variância, o coeficiente de variação, o coeficiente de assimetria e o coeficiente de curtose. $\mathrm{O}$ coeficiente de variação (CV) foi calculado com base no critério de Warrick \& Nielsen (1980), que classifica o CV como baixo $<12 \%$, médio de 12 a $24 \%$ e alto $>24 \%$. Para a hipótese de normalidade, foi utilizado o teste de Kolmogorov-Smirnov, disponível no software estatístico Minitab 14 (Minitab, 2000).

Para a caracterização da variabilidade espacial, utilizou-se a análise geoestatística. Sob a teoria da hipótese intrínseca, o semivariograma experimental foi estimado pela equação:

$$
\hat{\gamma}(\mathrm{h})=\frac{1}{2 \mathrm{~N}(\mathrm{~h})} \sum_{\mathrm{i}=1}^{\mathrm{N}(\mathrm{h})}\left[\mathrm{Z}\left(\mathrm{x}_{\mathrm{i}}\right)-\mathrm{z}\left(\mathrm{x}_{\mathrm{i}}+\mathrm{h}\right)\right]^{2}
$$

em que: $\gamma($ h) é o valor da semivariância para uma distância h; $\mathrm{N}(\mathrm{h})$, o número de pares envolvidos no cálculo da semivariância; Z(xi), o valor do atributo Z na posição xi; e Z(xi+h), o valor do atributo $Z$ separado por uma distância $h$ da posição xi.

Do ajuste de um modelo matemático aos valores calculados de $\hat{\gamma}$ (h) são definidos os coeficientes do modelo teórico para o semivariograma (efeito pepita,
$\mathrm{C}_{0}$; variância estrutural, $\mathrm{C}_{1}$; patamar, $\mathrm{C}_{0}+\mathrm{C}_{1}$; e alcance, a). $\mathrm{O}$ efeito pepita é o valor da semivariância para uma distância maior do que zero e menor do que a menor distância de amostragem e representa o componente da variação ao acaso; o patamar é o valor da semivariância em que a curva estabiliza sobre um valor constante; e o alcance é a distância da origem até onde o patamar atinge valores estáveis, expressando a distância além da qual as amostras não são correlacionadas (Trangmar et al., 1985).

Neste trabalho, utilizaram-se também os semivariogramas escalonados com o objetivo de reduzilos à mesma escala, facilitando a comparação entre resultados de diferentes variáveis. Os semivariogramas experimentais foram escalonados por meio da divisão das semivariâncias pela variância amostral de cada variável estudada (Guimarães, 1993; Vieira, 1997). Com as semivariâncias adimensionalizadas, o efeito pepita expressa diretamente em porcentagem do patamar (semivariância total) o componente aleatório da estrutura de variância. De acordo com Guimarães (1993), verifica-se proporcionalidade quando os semivariogramas escalonados permitem o ajuste de um único modelo para a variável em estudo.

Os semivariogramas experimentais escalonados foram ajustados ao modelo esférico (Equação 2).

$$
\left\{\begin{array}{l}
\hat{\gamma}(\mathrm{h})=\mathrm{C}_{0}+\mathrm{C}_{1}\left[\frac{3}{2}\left(\frac{\mathrm{h}}{\mathrm{a}}\right)-\frac{1}{2}\left(\frac{\mathrm{h}}{\mathrm{a}}\right)^{3}\right] \\
\hat{\gamma}(\mathrm{h})=\mathrm{C}_{0}+\mathrm{C}_{1}, \mathrm{se} \mathrm{h} \geq \mathrm{a}
\end{array}\right\}, \text { se } 0<\mathrm{h}<\mathrm{a}
$$

em que: $\mathrm{C}_{0}$ é o efeito pepita; $\mathrm{C}_{0}+\mathrm{C}_{1}$, o patamar; $\mathrm{h}$, a distância de separação entre duas observações; a, o alcance de dependência espacial.

Na determinação da existência ou não da dependência espacial (DE), utilizou-se o exame de semivariogramas, por meio do programa GS+ (Robertson, 1998). Em caso de dúvida entre mais de um modelo para o mesmo semivariograma, considerou-se o melhor coeficiente de determinação $\left(\mathrm{R}^{2}\right)$. Para analisar o grau da dependência espacial dos atributos em estudo, utilizou-se a classificação de Cambardella et al. (1994), em que são considerados dependência espacial forte os semivariogramas que têm efeito pepita menor ou igual a $25 \%$ do patamar, moderada quando está entre 25 e $75 \%$ e fraca quando for maior que $75 \%$.

\section{RESULTADOS E DISCUSSÕES}

Os resultados da estatística descritiva para os atributos físicos do solo estudados na área de floresta nativa e pastagem são apresentados no quadro 1. A média e mediana na área de floresta nativa e pastagem apresentaram valores próximos, mas ocorre distanciamento entre média e mediana nos atributos granulométricos e de estabilidade de agregados tanto em floresta nativa como na pastagem. 


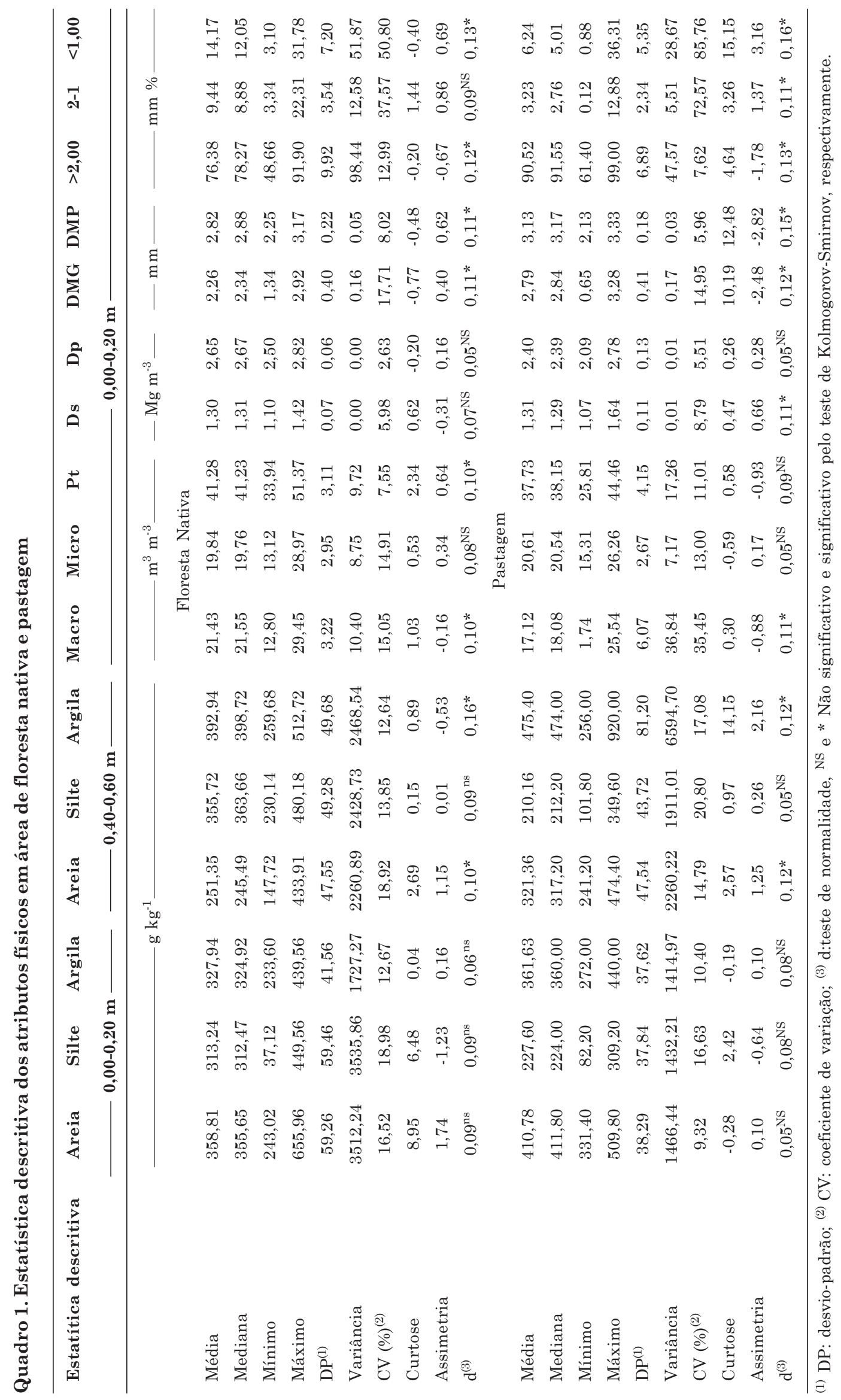


A assimetria e a curtose na área de floresta nativa e pastagem apresentaram valores negativos e positivos distantes do valor central zero e, conforme encontrado na média e mediana, a granulometria e a estabilidade de agregados foram os que mais se distanciaram do valor central zero. Estudo em geoestatística de Campos et al. $(2013 a, b)$ na região sul do Amazonas em área com agrofloresta encontrou valores distantes de zero em assimetria e curtose e da mesma forma com média e mediana dos atributos físicos. No entanto, segundo Guimarães et al. (2010), não caracterizam afastamento expressivo da normalidade, apenas evidenciam que, na natureza, não são encontradas distribuições que sejam absolutamente normais.

Segundo o critério de classificação de Warrick \& Nielsen (1980) para o coeficiente de variação (CV), a floresta nativa e pastagem tenderam a um mesmo comportamento de variabilidade, ocorrendo apenas alguns atributos que variaram na pastagem, quando comparado à floresta nativa. Os atributos físicos porosidade total $(\mathrm{Pt})$, densidade do solo $(\mathrm{Ds})$ e de partículas (Dp) e DMP foram classificados como baixos nas duas áreas; areia, argila e classe $>2,00$ na área de pastagem também apresentaram baixo CV; e já na floresta nativa apresentaram CV moderado. As classes de agregados 2,00-1,00 e >1,00 evidenciaram alto CV nas duas áreas e ainda a macroporosidade na pastagem também apresentou esse comportamento, se diferindo da floresta nativa que se apresenta com CV moderado.

Nesse sentido, observa-se que, apesar de o CV ser de baixo a alto, ocorre maior predominância de variabilidade moderada dos atributos físicos tanto no ambiente de floresta nativa como no de pastagem, indicando que nesses locais acontece moderada heterogeneidade. Oliveira et al. (2013) encontraram, na granulometria, CV baixo a alto no sul do Amazonas. Segundo Camargo et al. (2008), a medida estatística CV possibilita comparar a variabilidade entre amostras de variáveis com unidades diferentes, porém não permite analisar a variabilidade espacial dos atributos do solo nem seu padrão espacial.

Os atributos físicos apresentaram normalidade na sua maioria, sendo exceção à estabilidade de agregados (DMP, DMG , >2,00, 2,00-1,00, <1,00), macroporosidade e argila $(0,40-0,60 \mathrm{~m})$ nas duas áreas. Apesar de a normalidade não ser exigência na geoestatística, essa permite observar maior precisão das estimativas da krigagem por meio de seus valores médios (Cressie, 1991).

$\mathrm{Na}$ estatística descritiva, é possível verificar pequena variação nos atributos físicos em solo de pastagem em relação ao da floresta nativa, tanto nos valores da média, mediana, assimetria e curtose quando apresentam distanciamentos maiores que o da floresta nativa quanto no coeficiente de variação, em que é possível verificar maiores variações em relação à floresta nativa. Sendo assim, o uso da geoestatística para avaliar a variabilidade dos atributos pela análise da dependência espacial vem facilitar o entendimento do padrão de ocorrência desses atributos.

Os resultados referentes à análise geoestatística encontram-se no quadro 2. As variáveis DMG, DMP e classe $<1,00$, na área de floresta nativa, e silte $(0,40$ $0,60 \mathrm{~m}$ ), na área de pastagem, não apresentaram dependência espacial; nesse caso, encontram-se com distribuição aleatória (efeito pepita puro) para distâncias maiores que a menor distância de amostragem, que foi de $10 \mathrm{~m}$, distribuindo-se de forma independente no espaço, indicando que para essas variáveis a distância de amostragem não foi capaz de exibir toda a variância dos dados (Carvalho et al., 2011). Nesse caso, o uso da geoestatística não é recomendado, sendo mais indicado o uso da estatística clássica (Souza et al., 2004).

Os atributos estudados foram submetidos à aplicação de semivariograma, com a finalidade de avaliar sua dependência espacial. O modelo de semivariograma esférico foi o que predominou nas duas áreas estudadas. Segundo Isaaks \& Srivastava (1989), os modelos esféricos descrevem propriedades com alta continuidade espacial, ou menos erráticos na curta distância.

Observou-se um comportamento diferente dos parâmetros geoestatísticos nos ambientes em estudo. No geral, os modelos ajustados apresentaram valores bem variáveis de efeito pepita da floresta nativa, em relação à pastagem. $\mathrm{O}$ menor valor de efeito pepita (EP) na floresta nativa da granulometria é 0,00 , enquanto na área de pastagem a variação foi muito elevada, em que o menor valor apresentado foi 437. Isso refletiu em maior descontinuidade entre as amostras, conforme afirmação de Vieira (2000), pois quanto menor a proporção do efeito pepita em relação ao patamar do semivariograma, maior será a dependência espacial apresentada pelo atributo que reflete em maior continuidade do fenômeno, menor a variância da estimativa e maior confiança no valor estimado.

Verificou-se na área de pastagem um comportamento muito próximo ao da floresta nativa, onde os atributos se apresentaram com grau de dependência espacial (DE) moderada e fraca. $\mathrm{Na}$ pastagem, observou-se maior presença de DE fraca, indicando que esses locais podem estar sofrendo maior pressão de fatores extrínsecos como tráfego de maquinaria agrícola, pisoteio de animais, chuva intensa etc., possivelmente em razão a sua maior exposição em relação à floresta nativa. Campos et al. $(2013 a, b)$ encontraram DE moderada na maioria dos atributos físicos estudados na região sul do Amazonas.

Uma maneira também de avaliar a variabilidade espacial dos atributos do solo por meio da geoestatística é utilizando os semivariogramas escalonados (Figura 2). O modelo esférico ajustou-se a todos os semivariogramas escalonados na área de floresta nativa e pastagem, seguindo, nesse caso, o mesmo comportamento apresentado nos resultados dos 
semivariogramas individuais e ainda concordando com afirmações de Grego \& Vieira (2005), de que esse modelo é o que predomina nos trabalhos em ciência do solo.

O EP apresentou valores bem próximos entre floresta nativa e pastagem, diferentemente dos apresentados nos semivariogramas simples, em que se verificou grande discrepância entre os atributos de cada área. Já a dependência espacial foi classificada como moderada nas duas áreas, seguindo os resultados dos semivariogramas individuais e estando de acordo com os resultados encontrados por Mercante et al. (2003) e Silva Cruz et al. (2010). O alcance apresentou valores bem superiores ao evidenciado nos semivariogramas individuais nas duas áreas onde o menor valor foi de $30 \mathrm{~m}$.

Pôde-se observar que na área de floresta nativa os atributos físicos se encontram mais próximos na camada de 0,00-0,20 m, comparado aos atributos físicos na área de pastagem que se apresentam dispersos, indicando diferentes padrões de variabilidade espacial para as variáveis analisadas.

Apesar de ter ocorrido em algumas variáveis de floresta nativa e pastagem a não ocorrência de dependência espacial, a maior parte dos atributos

Quadro 2. Modelos e parâmetros estimados dos semivariogramas dos atributos físicos em área de floresta nativa

\begin{tabular}{|c|c|c|c|c|c|c|c|}
\hline Atributo físico & Prof. & Modelo $^{(1)}$ & $\mathbf{E P}^{(2)}$ & Patamar & Alcance & D.E..$^{(3)}$ & $\mathbf{R}^{2}$ \\
\hline & \multicolumn{7}{|l|}{$\mathrm{cm}$} \\
\hline & \multicolumn{7}{|c|}{ Floresta nativa } \\
\hline Areia & $0,00-0,20$ & Exp. & 0,00 & 0,02 & 18,90 & 63 & 0,59 \\
\hline Silte & $0,00-0,20$ & Exp. & 0,03 & 0,10 & 44,56 & 61 & 0,92 \\
\hline Argila & $0,00-0,20$ & Esf. & 0,00 & 0,01 & 17,30 & 81 & 0,94 \\
\hline Areia & $0,40-0,60$ & Esf. & 0,00 & 0,01 & 21,00 & 88 & 0,77 \\
\hline Silte & $0,40-0,60$ & Exp. & 0,00 & 0,02 & 27,60 & 71 & 0,85 \\
\hline Argila & $0,40-0,60$ & Exp. & 0,00 & 0,01 & 14,70 & 73 & 0,61 \\
\hline Macro & $0,00-0,20$ & Esf. & 1,17 & 11,57 & 15,70 & 89 & 0,91 \\
\hline Micro & $0,00-0,20$ & Esf. & 0,00 & 0,02 & 36,30 & 62 & 0,90 \\
\hline $\mathrm{Pt}$ & $0,00-0,20$ & Esf. & 7,25 & 2,15 & 14,30 & 66 & 0,94 \\
\hline Ds & $0,00-0,20$ & Esf. & 2,00 & 3,86 & 14,50 & 94 & 0,91 \\
\hline $\mathrm{Dp}$ & $0,00-0,20$ & Exp. & 2,10 & 6,97 & 12,00 & 69 & 0,64 \\
\hline DMG & $0,00-0,20$ & $\mathrm{EPP}$ & - & - & - & - & - \\
\hline DMP & $0,00-0,20$ & EPP & - & - & - & - & - \\
\hline$>2,00 \mathrm{~mm}$ & $0,00-0,20$ & Exp. & 0,00 & 0,01 & 13,50 & 68 & 0,80 \\
\hline $2,00-1,00 \mathrm{~mm}$ & $0,00-0,20$ & Esf. & 0,02 & 0,13 & 15,90 & 80 & 0,92 \\
\hline \multirow[t]{2}{*}{$<1,00 \mathrm{~mm}$} & $0,00-0,20$ & EPP & - & - & - & - & - \\
\hline & \multicolumn{7}{|c|}{ Pastagem } \\
\hline Areia & $0,00-0,20$ & Exp. & 605,81 & 1262,49 & 40,20 & 52 & 0,83 \\
\hline Silte & $0,00-0,20$ & Esf. & 574,19 & 1504,00 & 12,90 & 61 & 0,60 \\
\hline Argila & $0,00-0,20$ & Exp. & 437,17 & 1324,00 & 14,70 & 66 & 0,67 \\
\hline Areia & $0,40-0,60$ & Exp. & 718,00 & 2390,00 & 33,00 & 70 & 0,97 \\
\hline Silte & $0,40-0,60$ & $\mathrm{EPP}$ & - & - & - & - & - \\
\hline Argila & $0,40-0,60$ & Esf. & 1406,96 & 6504,00 & 15,70 & 78 & 0,86 \\
\hline Macro & $0,00-0,20$ & Exp. & 2,27 & 13,31 & 18,30 & 82 & 0,66 \\
\hline Micro & $0,00-0,20$ & Esf. & 1,67 & 3,62 & 52,30 & 53 & 0,98 \\
\hline $\mathrm{Pt}$ & $0,00-0,20$ & Esf. & 1,22 & 9,91 & 18,00 & 87 & 0,86 \\
\hline Ds & $0,00-0,20$ & Esf. & 0,00 & 0,01 & 29,70 & 83 & 0,73 \\
\hline $\mathrm{Dp}$ & $0,00-0,20$ & Esf. & 0,00 & 0,01 & 12,80 & 74 & 0,75 \\
\hline DMG & $0,00-0,20$ & Esf. & 0,04 & 0,18 & 15,90 & 76 & 0,94 \\
\hline DMP & $0,00-0,20$ & Esf. & 0,00 & 0,03 & 18,80 & 80 & 0,98 \\
\hline$>2,00 \mathrm{~mm}$ & $0,00-0,20$ & Exp. & 15,73 & 48,98 & 22,50 & 67 & 0,90 \\
\hline $2,00-1,00 \mathrm{~mm}$ & $0,00-0,20$ & Esf. & 0,39 & 2,53 & 15,10 & 84 & 0,81 \\
\hline$<1,00 \mathrm{~mm}$ & $0,00-0,20$ & Esf. & 5,84 & 30,64 & 19,10 & 80 & 0,97 \\
\hline
\end{tabular}

(1) EPP: Efeito Pepita Puro; Exp.: exponencial; e Esf.: Esférico; ${ }^{(2)}$ EP: Efeito Pepita; ${ }^{(3)}$ D.E.: grau de dependência espacial $\left(\left[\mathrm{C}_{0} /\left(\mathrm{C}_{0}+\mathrm{C}_{1}\right)\right] \times 100\right)$. 

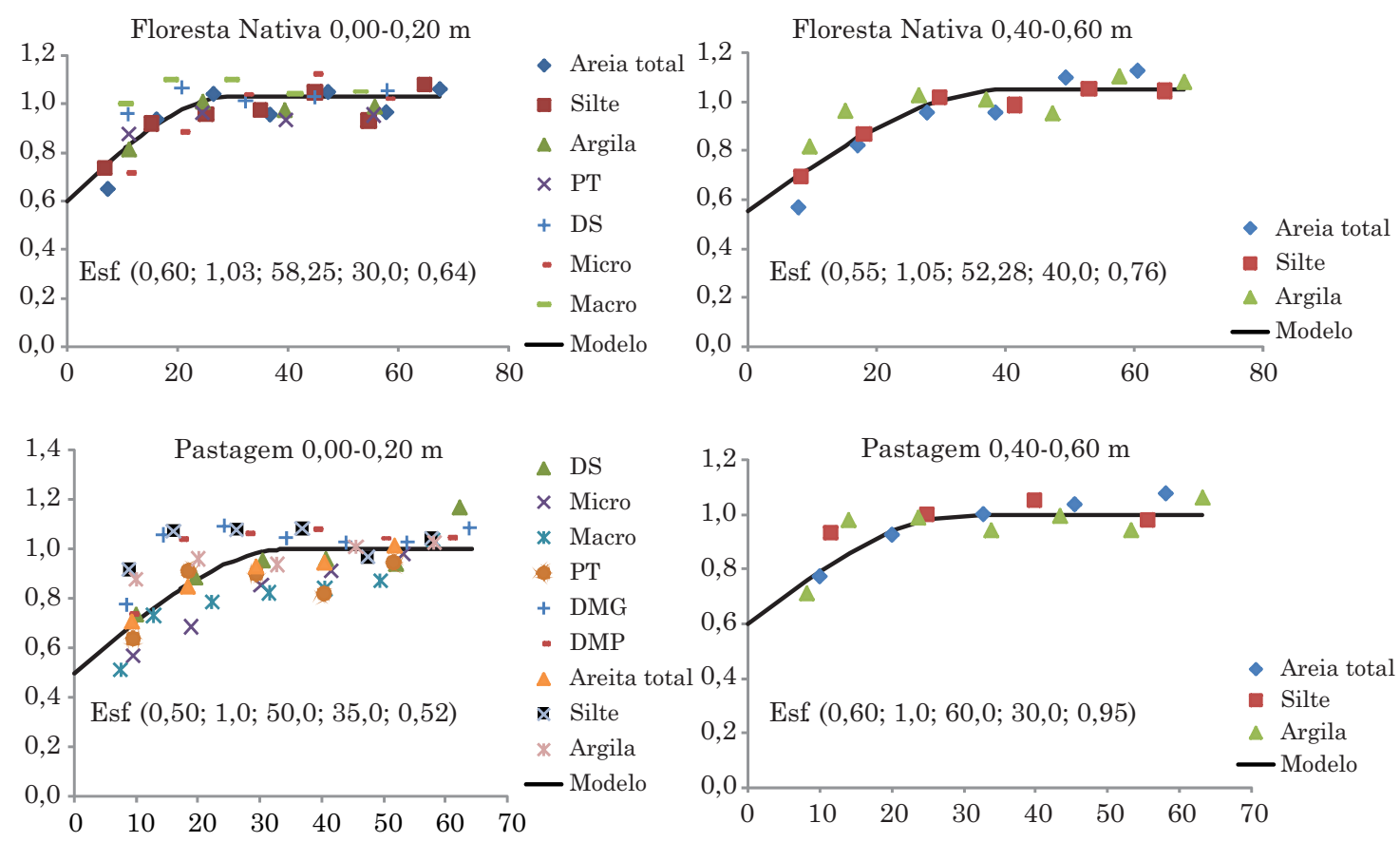

Figura 2. Semivariogramas escalonados para os atributos físicos em área de floresta nativa e pastagem na região sul do Amazonas.

apresentaram dependência espacial demonstrando que os semivariogramas explicam a maior parte da variância dos dados experimentais. Nesse sentido, Souza et al. (2009) afirmaram que uma das principais utilizações dos semivariogramas é na determinação do número ideal de amostras para a estimação das características do solo.

O alcance é uma medida importante no planejamento e na avaliação experimental, já que pode auxiliar na definição de procedimento de amostragem (McBratney \& Webster, 1986). Esse parâmetro representa a distância em que os pontos amostrais estão correlacionados. Com o conhecimento do alcance da dependência espacial, define-se o raio de amostragem, em que os atributos estudados apresentaram diferentes valores de alcance em ambas as áreas, mas com valores acima do estipulado pela malha. Com esses dados, possibilitaram estimar valores médio de densidade amostral de 35 pontos/ha e espaçamento de $20,61 \mathrm{~m}$ na área de floresta nativa, enquanto na área de pastagem o valor médio de densidade amostral foi de 29,80 pontos/ ha e espaçamento de 22,93 m (Quadro 3).

Esses resultados obtidos pelos valores de alcance de cada variável, em que se obtêm o valor médio de densidade amostral e o espaçamento, na prática estão indicando que nesses locais a densidade amostral ideal na área de floresta é de 35 pontos/ha com espaçamento entre esses pontos de 20,61 m; na área de pastagem a densidade amostral ideal é de 29,80 pontos/ha com espaçamentos de 22,93 m (Quadro 3).

Pôde-se observar que os valores de densidade amostral e o espaçamento com base no alcance apresentaram valores bem abaixo ao usado neste trabalho e que na área de floresta nativa o número de pontos necessários para a avaliação do solo ideal é maior que o apresentado na área de pastagem, evidenciando que, mesmo estando em áreas paralelas com uma distância próxima e mesmo solo, a retirada da floresta para outros fins resulta em alteração na variabilidade natural dos atributos do solo. Essas informações podem viabilizar o trabalho no campo, uma vez que estudos de amostragem têm sido conduzidos visando reduzir a variabilidade das características do solo (Montanari et al., 2005).

\section{CONCLUSÕES}

1. A pastagem apresentou leve variação nos seus atributos físicos em relação à floresta nativa, com maior presença de coeficiente de variação alto e dependência espacial fraca.

2. Os semivariogramas escalonados conseguiram reproduzir de forma satisfatória o comportamento espacial dos atributos no mesmo padrão dos semivariogramas individuais, possibilitando seu uso para estimar a variabilidade natural dos atributos do solo.

3. O uso do parâmetro alcance do semivariograma evidenciou-se eficiente para determinar a densidade amostral ideal para os ambientes em estudo, podendo esses resultados servir como base para futuros estudos na região sul do Amazonas para levantamentos de solos. 
Quadro 3. Valores de densidade amostral e espaçamento ideal com base no alcance estimado na análise geoestatística em área de floresta nativa e pastagem

\begin{tabular}{|c|c|c|c|c|}
\hline \multirow{3}{*}{ Atributo físico } & \multicolumn{4}{|c|}{ Planejamento amostral - Geoestatística } \\
\hline & \multicolumn{2}{|c|}{ Floresta Nativa } & \multicolumn{2}{|c|}{ Pastagem } \\
\hline & Densidade amostral & Espaçamento & Densidade amostral & Espaçamento \\
\hline & pontos/ha & $\mathrm{m}$ & pontos/ha & $\mathrm{m}$ \\
\hline Areia $(0,00-0,20 \mathrm{~m})$ & 28 & 19 & 6 & 40 \\
\hline Silte $(0,00-0,20 \mathrm{~m})$ & 5 & 45 & 60 & 13 \\
\hline Argila $(0,00-0,20 \mathrm{~m})$ & 33 & 17 & 46 & 15 \\
\hline Areia $(0,40-0,60 \mathrm{~m})$ & 23 & 21 & 9 & 33 \\
\hline Silte $(0,40-0,60 \mathrm{~m})$ & 13 & 28 & - & - \\
\hline Argila $(0,40-0,60 \mathrm{~m})$ & 46 & 15 & 41 & 16 \\
\hline Macroporosidade & 41 & 16 & 30 & 18 \\
\hline Microporosidade & 8 & 36 & 4 & 52 \\
\hline Porosidade total & 49 & 14 & 31 & 18 \\
\hline Densidade do solo & 48 & 15 & 11 & 30 \\
\hline Densidade de partículas & 69 & 12 & 61 & 13 \\
\hline Diâmetro médio geométrico & - & - & 20 & 22 \\
\hline Diâmetro médio ponderado & - & - & 36 & 17 \\
\hline$>2,00$ & 55 & 14 & 28 & 19 \\
\hline $2,0-1,0 \mathrm{~mm}$ & 40 & 16 & 20 & 23 \\
\hline$<1,00 \mathrm{~mm}$ & - & - & 44 & 15 \\
\hline Média & 35 & 20,61 & 29,80 & 22,93 \\
\hline
\end{tabular}

4. Os resultados geoestatísticos expressos pelos semivariogramas individuais e escalonados indicaram que a retirada da floresta nativa para a implantação da pastagem modifica a variabilidade natural dos atributos físicos.

\section{AGRADECIMENTOS}

À FAPEAM e ao SECT-AM, pelo financiamento da pesquisa.

\section{LITERATURA CITADA}

ALENCAR, A.; NEPSTAD, D.; McGRATH, D.; MOUTINHO, P.; PACHECO, P.; DIAZ, M. C.V. \& SOARES FILHO, B.S. Desmatamento na Amazônia: Indo além da "emergência crônica”. Belém, Instituto de Pesquisa Ambiental da Amazônia, 2004. 85p.

BECKER, B.K. Geopolítica da Amazônia. Est. Avançados, 19:71-86, 2005.

BRASIL. Ministério das Minas e Energia. Projeto Radambrasil, folha SB. 20, Purus. Rio de Janeiro, 1978. 561p.

CAMARGO, L.A.; MARQUES JÚNIOR, J.; PEREIRA, G.T. \& HORVAT, R.A. Variabilidade espacial de atributos mineralógicos de um Latossolo sob diferentes formas do relevo. II - Correlação espacial entre mineralogia e agregados. R. Bras. Ci. Solo, 32:2279-2288, 2008.
CAMBARDELLA, C.A.; MOORMAN, T.B.; NOVAK, J.M.; PARKIN, T.B.; KARLEN, D.L.; TURCO, R.F. \& KONOPKA, A.E. Field-scale variability of soil properties in Central Iowa. Soil Sci. Soc. Am. J., 58:1501-1511, 1994.

CAMPOS, M.C.C.; AQUINO, E.A.; OLIVEIRA, I.A. \& BERGAMIN, A.C. Variabilidade espacial da resistência mecânica do solo à penetração e umidade do solo em área cultivada com cana-de-açúcar na região de Humaitá, Amazonas, Brasil. Agrária, 8:305-310, $2013 \mathrm{~b}$.

CAMPOS, M.C.C.; OLIVEIRA, I.A.; SANTOS, L.A.C.; AQUINO, E.A. \& SOARES, M.D.R. Variabilidade espacial da resistência do solo à penetração e umidade em áreas cultivadas com mandioca na região de Humaitá, AM. R. Agro@mbi., 6:9-16, 2012.

CAMPOS, M.C.C.; SOARES, M.D.R.; SANTOS, L.A.C.; OLIVEIRA, I.A. \& AQUINO, E.A. Spatial variability of physical attributes in Alfissol under agroforestry, Humaitá region, Amazonas state, Brazil. R. Ci. Agrar., 56:149-159, 2013a.

CARDOSO, E.L.; SILVA, M.L.N.; MOREIRA, F.M.S. \& CURI, N. Atributos biológicos indicadores da qualidade do solo em pastagem cultivada e nativa no Pantanal. Pesq. Agropec. Bras., 44:631-637, 2009.

CARVALHO, L.A.; MEURER, I.; SILVA JUNIOR, C.A.; CAVALIERI, K.M.V. \& SANTOS, C.F.B. Dependência espacial dos atributos físicos de três classes de solos cultivados com cana-de-açúcar sob colheita mecanizada. Agriambi, 15:940-949, 2011. 
CAVAlCANTE, E.G.S.; ALVES, M.C.; SOUZA, Z.M. \& PEREIRA, G.T. Variabilidade espacial de atributos químicos do solo sob diferentes usos e manejos. R. Bras. Ci. Solo, 31:1329-1339, 2007.

COELHO, E.C.; SOUZA, E.G.; URIBE-OPAZO, M.A. \& PINHEIRO NETO, R. Influência da densidade amostral e do tipo de interpolador na elaboração de mapas temáticos. Acta Sci., 31:165-174, 2009.

CRESSIE, N. Statistics for spatial data. New York, John Wiley, 1991. 920p.

EMPRESA BRASILEIRA DE PESQUISA AGROPECUÁRIA EMBRAPA. Centro Nacional de Pesquisa de Solos. Manual de métodos de análise de solo. Rio de Janeiro, 1997. 212p.

EMPRESA BRASILEIRA DE PESQUISA AGROPECUÁRIA EMBRAPA. Centro Nacional de Pesquisa de Solos. Sistema brasileiro de classificação de solos. Brasília, 2006. $306 \mathrm{p}$.

GREGO, C.R. \& VIEIRA, S.R. Variabilidade espacial de propriedades físicas do solo em uma parcela experimental. R. Bras. Ci. Solo, 29:169-177, 2005.

GUIMARÃES, E.C. Variabilidade espacial da umidade e da densidade do solo em um Latossolo Roxo. Campinas, Universidade de Campinas, 1993. 138p. (Dissertação de Mestrado)

GUIMARÃES, R.M.L.; GONÇALVES, A.C.A.; TORMENA, C.A.; FOLEGATTI, M.V. \& BLAINSKI, E. Variabilidade espacial de propriedades físico-hídricas de um Nitossolo sob a cultura do feijoeiro irrigado. Eng. Agríc., 30:657-669, 2010.

ISAAKS, E.H. \& SRIVASTAVA, R.M. An introduction to applied geoestatistics. New York, Oxford University Press, 1989. $561 \mathrm{p}$.

KEMPER, W.D. \& CHEPIL, W.S. Aggregate stability and size distribution. In: BLACK, C.A., ed. Methods of soil analysis. Madison, American Society of Agronomy, 1965. p.499510.

KEMPER, W.D. \& ROSENAU, R.C. Aggregate stability and size distribution, In: KLUTE, A., ed. Methods of soil analysis. 2.ed. Madison, American Society of Agronomy, 1986. p.425-441.

LARK, R.M. Designing sampling grids from imprecise information on soil variability, an approach based on the fuzzy kriging variance. Geoderma, 98:35-39, 2000.

LIMA, J.S.S.; SATTLER, M.A.; PASSOS, R.R.; OLIVEIRA II, P.C. \& SOUZA, G.S. Variabilidade espacial de atributos físicos de um Argissolo Vermelho-Amarelo sob pastagem e vegetação secundária em Regeneração natural. Eng. Agríc., 29:185-195, 2009.

McBRATNEY, A.B. \& WEBSTER, R. Choosing functions for semi-variograms of soil properties and fitting them to sampling estimates. J. Soil Sci., 37:p.617-639, 1986.

MERCANTE, E.; URIBE-OPAZO, M.A. \& SOUZA, E.G. Variabilidade espacial e temporal da resistência mecânica do solo à penetração em áreas com e sem manejo químico localizado. R. Bras. Ci. Solo, 27:1149-1159, 2003.
MINITAB Release 14.1. Statistical Software. US/Canadá, 2000.

MONTANARI, R.; MARQUES JÚNIOR, J.; PEREIRA, G.T. \& SOUZA, Z.M. Forma da paisagem como critério para otimização amostral de Latossolos sob cultivo de canade-açúcar. Pesq. Agropec. Bras., 40:69-77, 2005.

OLIVEIRA, I.A.; CAMPOS, M.C.C.; SOARES, M.D.R.; AQUINO, R.E.; MARQUES JÚNIOR, J. \& NASCIMENTO, E.P. Variabilidade espacial de atributos físicos em um Cambissolo Háplico, sob diferentes usos na região Sul do Amazonas. R. Bras. Ci. Solo, 37:11031112, 2013.

ROBERTSON, G.P. GS+ geostatistics for the environmental sciences: GS+ user's guide. Plainwell, Gamma Design Software, 1998. 152p.

SILVA CRUZ, J.; ASSIS JÚNIOR, R.N.; MATIAS, S.S.R.; CAMACHO-TAMAYO, J.H. \& TAVARES, R.C. Análise espacial de atributos físicos e carbono orgânico em Argissolo Vermelho-Amarelo cultivado com cana-deaçúcar. Ci. Agrotéc., 34:271-278, 2010.

SILVA NETO, S.P.; SANTOS, A.C.; LEITE, R.L.L.; DIM, V.P.; NEVES NETO, D.N. \& SILVA, J.E.C. Variação espacial do teor de matéria orgânica do solo e produção de gramínea em pastagens de capim-marandu. Biosci. J., 28:41-53,2012.

SOUZA, Z.M.; MARQUES JÚNIOR, J. \& PEREIRA, G.T. Geoestatística e atributos do solo em áreas cultivadas com cana-de-açúcar. Ci. Rural, 40:48-56, 2009.

SOUZA, Z.M.; MARQUES JÚNIOR, J.; PEREIRA, G.T. \& BENTO, M.J.C. Variabilidade espacial de atributos físicos de um Latossolo Vermelho sob cultivo de cana-de-açúcar. R. Bras. Eng. Agríc. Amb., 8:51-58, 2004.

SOUZA, Z.M.; MAQUES JUNIOR, J.; PEREIRA, G.T. \& MONTANARI, R. Otimização amostral de atributos de Latossolos considerando aspectos solo-relevo. Ci. Rural, 36:826-836, 2006b.

SOUZA, Z.M.; MARQUES JÚNIOR, J.; PEREIRA, G.T.; MONTANARI, R. \& CAMPOS, M.C.C. Amostragem de solo para determinação de atributos químicos e físicos em área com variação nas formas do relevo. Científica, 34:249-256, 2006a.

TRANGMAR, B.B.; YOST, R.S. \& UEHARA, G. Application of geostatistics to spatial studies of soil properties. Adv. Agron., 38:54-94, 1985.

VIEIRA, S.R. Geoestatística em estudo da variabilidade espacial do solo. In: NOVAIS, R.F.; ALVAREZ, V., V.H. \& SCHAEFER, G.R., eds. Tópicos em ciência do solo. Viçosa, MG, Sociedade Brasileira de Ciência do Solo, 2000. v.1, p.1-54.

VIEIRA, S.R. Variabilidade espacial de argila, silte e atributos químicos em uma parcela experimental de um Latossolo Roxo de Campinas (SP). Bragantia, 56:181-190,1997.

WARRICK, A.W. \& NIELSEN, D.R. Spatial variability of soil physical properties in the field. In: HILLEL, D., ed. Applications of soil physics. New York, 1980. p. 319-344. 